\title{
EFEITOS COLATERAIS DE MEDICAMENTOS UTILIZADOS POR PACIENTES ONCOLÓGICOS E SUA RELAÇÃO COM O ESTADO NUTRICIONAL MEDICAMENTOS E ESTADO NUTRICIONAL
}

\author{
Maithe Alves De Lima, Maisa Marchiori Bernusse, Sandra Cristina Genaro. \\ Universidade do Oeste Paulista - UNOESTE, Faculdade de Nutrição, Presidente Prudente, SP.
}

\begin{abstract}
RESUMO
O impacto global do câncer dobrou em 30 anos. Essa doença bem como seu tratamento é responsável pela redução da qualidade de vida e maior morbimortalidade. Esse estudo trata-se de uma pesquisa transversal de natureza descritiva, cujo objetivo foi observar a relação dos efeitos colaterais dos medicamentos, com o estado nutricional de 99 pacientes com câncer em tratamento quimioterápico. Os dados foram submetidos ao teste de correlação de Spearman e realizados no programa SPSS 21.0, sendo considerado como significante com o $p<0,05$. Os sintomas que causaram perda de peso foram os relacionados à anorexia, disfagia e enterite. A mucosite apresentou relação inversa com a perda de peso. É imprescindível conhecer os efeitos colaterais dos tratamentos antineoplásicos, principalmente os quimioterápicos, para que, com ações preventivas, possam-se minimizar os sintomas, a fim de evitar ou reduzir a depleção do estado nutricional do paciente, responsável por um pior prognóstico.
\end{abstract}

Palavras-chave: Estado nutricional. Neoplasia maligna. Sintomas. Desnutrição. Tratamento.

\section{SIDE EFFECTS OF MEDICATIONS USED BY CANCER PATIENTS AND THEIR RELATION TO NUTRITIONAL STATUS}

\begin{abstract}
The global impact of cancer has doubled in 30 years. This disease as well as its treatment is responsible for the reduction of the quality of life and greater morbimortality. This study is a descriptive cross-sectional study whose objective was to observe the relationship between the side effects of the drugs and the nutritional status of 99 cancer patients on chemotherapy. The data were submitted to the Spearman correlation test and performed in the SPSS 21.0 program, being considered significant with $p<0.05$. The symptoms that caused weight loss were related to anorexia, dysphagia and enteritis. Mucositis presented an inverse relationship with weight loss. It is imperative to know the side effects of antineoplastic treatments, especially chemotherapy, so that, with preventive actions, symptoms can be minimized in order to avoid or reduce the depletion of the patient's nutritional status, which is responsible for a worse prognosis.
\end{abstract}

Keywords: Nutritional status. Malignant neoplasm. Symptoms. Malnutrition. Treatment. 


\section{INTRODUÇÃO}

Um relatório da Agência Internacional para Pesquisa em Câncer (IARC) e Organização Mundial da Saúde (OMS) revelou que o impacto global do câncer dobrou em 30 anos $^{1}$. Atualmente mais de 32 milhões de pessoas vivem com câncer em todo mundo e para o biênio 2016/2017, a estimativa é que surjam 596.070 novos casos no Brasil, sendo 295.200 em homens e 300.870 em mulheres $^{2}$. É esperado que nas próximas décadas, o impacto desta enfermidade na população, corresponda a $80 \%$ dos mais de 4 milhões de casos novos estimados para 2025, impulsionado em parte pelo envelhecimento da população e mudança de estilos de vida ${ }^{3}$.

Essa doença é capaz de causar grave perda de peso ao paciente, sendo que algumas de suas causas é o hipermetabolismo, além do aumento da leptina que leva à diminuição do apetite, induzindo à caquexia ${ }^{4}$. Essa perda grave de peso é responsável pelo mal prognostico com redução da qualidade de vida, maior morbidade e mortalidade ${ }^{5}$.

Os resultados dos tratamentos convencionais são promissores, porém podem causar desconfortos no sistema digestório, capazes de reduzir a ingestão alimentar e consequentemente causar depleção do estado nutricional ${ }^{6}$.

Em contrapartida, o aumento de peso ponderal pode ser observado em alguns pacientes oncológicos cujas drogas quimioterápicas são responsáveis por retenção hídrica e indução ao aumento do apetite ${ }^{7}$.

Com a utilização da quimioterapia, alguns pacientes podem apresentar sintomas que apresentam alteração na ingestão alimentar e redução na absorção de nutrientes. Esse fato é capaz de comprometer o estado nutricional e imunológico, podendo ser responsável por uma possível interrupção do tratamento proposto. Neste contexto, o referente trabalho buscou observar a relação dos efeitos colaterais dos medicamentos utilizados em pacientes com câncer, em tratamento quimioterápico, com seu estado nutricional.

\section{MÉTODOS}

Este trabalho foi encaminhado ao Comitê de Ética e Pesquisa, seguindo as normas da Resolução CNS 466/2012 do CONEP, pela Universidade do Oeste Paulista e Hospital Regional de Presidente Prudente, SP.

Tratou-se de uma pesquisa transversal de natureza descritiva, nos meses de fevereiro de 2015 a setembro de 2016, realizada em 63 pacientes, portadores de câncer, do setor de oncologia de um hospital público e 36 pacientes pertencentes a uma clínica de oncologia; ambos locais situados na cidade de Presidente Prudente, SP.

Para avaliação do estado nutricional, utilizou-se a classificação pelo IMC de acordo com os critérios da OMS (1997) ${ }^{8}$ para adultos e Lipschitz (1994) $)^{9}$ para idosos ( $\geq 60$ anos). Embora esse método não seja a melhor forma para avaliar o perfil nutricional do paciente oncológico, foi utilizado nesse estudo por ser um método fácil e rápido de ser utilizado e por não haver disponível materiais e equipamentos adequados para outra forma de avaliação.

Analisou-se também a perda de peso involuntária do paciente do estudo, utilizando o critério apresentado por Blackburn e Bistrian $(1977)^{10}$. Este dado é importante para avaliar o estado de saúde do paciente, já que possui correlação com a mortalidade.

Para verificação do diagnóstico e dados relacionados à história clínica (tipo de tumor, estadiamento), bem como quimioterápicos e medicamentos utilizados, foi realizada uma busca aos prontuários dos pacientes.

As análises estatísticas foram realizadas no programa SPSS 21.0, utilizando teste de correlação de Spearman, sendo considerado como estatisticamente significante quando a probabilidade for menor a 0,05 ( $p$-valor $<0,05$ ). 


\section{RESULTADOS}

Dos pacientes do estudo, $48,5 \%$ eram do sexo feminino e $51,5 \%$ do sexo masculino. Do total, 54\% encontravam-se na faixa etária de 56 a 75 anos.

O câncer mais prevalente na população estudada, independente do sexo, foi o câncer de Sistema Digestório (estômago, cólon, reto e esôfago) (29,3\%), seguido pelo câncer de mama $(20,2 \%)$, Hematológico $(10,1 \%)$, Cabeça e Pescoço $(9,1 \%)$, Próstata $(8,1 \%)$, Pulmão $(7,1 \%)$, Fígado $(7,1 \%)$, Útero $(6,1 \%)$, Pele $(2,0 \%)$, outros $(1,0 \%)$.

De acordo com a classificação do IMC, observou-se que a maioria dos pacientes encontravam-se eutróficos $(43,4 \%)$ e $35,4 \%$ com sobrepeso. Com relação à obesidade, $12,1 \%$ apresentavam obesidade grau I; $2 \%$, grau II e $3 \%$ grau III. Somente $4,1 \%$ foram classificados com desnutrição grau I.

Mesmo apresentando uma maior porcentagem de pacientes eutróficos, sobrepeso ou obesos, verificou-se a presença de perda de peso involuntária nesses pacientes, demonstrada conforme a tabela 1 .

Tabela 1. Análise da perda de peso

(Tempo de perda de peso - Média \pm Desvio padrão: $11 \pm 12$ dias)

(\% de perda de peso - Média \pm Desvio padrão: 6,3 \pm 7,5\%)

$\%$

(Peso habitual - Média \pm Desvio padrão: 74,1 $\pm 17,5 \mathrm{~kg}$ )

Sem perda de peso

39,4

Aceitável

32,3

Perda de peso significativa

6,1

Perda de peso grave

22,2

Total

100,0

Muitos são os efeitos colaterais causados por medicamentos, principalmente os relacionado aos sintomas gastrointestinais, responsáveis pela diminuição da ingestão alimentar e má absorção, tendo como consequência a perda de peso, acarretando depleção do estado nutricional.

Com a presença dos sintomas, as modificações na consistência da dieta foram inevitáveis. Dos pacientes da pesquisa, 21,3\% apresentaram alteração na consistência da dieta sendo capaz de prejudicar o aporte calórico e proteico, podendo contribuir ainda mais para a perda de peso dos pacientes.

Nesse estudo verificaram-se efeitos colaterais de 41 drogas utilizadas pelos pacientes. Dentre elas, as que estavam relacionadas à perda ponderal significativa ou grave, foi anorexia, disfagia e enterite. Já a mucosite apresentou relação inversa neste trabalho, ou seja, a presença da mucosite apresentou evidência de relação com a diminuição do percentual de perda de peso, como observado na Tabela 2. 
Tabela 2. Relação dos sintomas com o peso

\begin{tabular}{|c|c|c|}
\hline Variável & Estatística $\rho$ (rho) de Spearman & p-valor \\
\hline Alteração apetite & $-0,192$ & 0,057 \\
\hline Alteração do Paladar & 0,006 & 0,954 \\
\hline Alteração na Consistência da dieta & $-0,152$ & 0,134 \\
\hline Anemia & $-0,161$ & 0,111 \\
\hline Anorexia & $-0,213$ & $0,001 *$ \\
\hline Cáries & $-0,099$ & 0,328 \\
\hline Constipação & $-0,151$ & 0,135 \\
\hline Diarreia & $-0,106$ & 0,297 \\
\hline Disfagia & $-0,211$ & $0,036^{*}$ \\
\hline Enterite & $-0,295$ & $0,003 *$ \\
\hline Estomatite & 0,014 & 0,890 \\
\hline Gases & 0,026 & 0,799 \\
\hline Leucopenia & 0,090 & 0,377 \\
\hline Mucosite & 0,199 & $0,049 *$ \\
\hline Mudanças nas Preferências Alimentares & $-0,127$ & 0,211 \\
\hline Náuseas & $-0,005$ & 0,959 \\
\hline Vômitos & $-0,136$ & 0,179 \\
\hline Xerostomia & 0,044 & 0,667 \\
\hline
\end{tabular}

* Correlação de Spearman estatisticamente significativa ao nível de significância de 5\% $(0,05)$.

\section{DISCUSSÃO}

Os tumores localizados nas regiões da cabeça, pescoço, pulmão, esôfago, estômago, cólon, reto, fígado e pâncreas possuem maior influência na desnutrição. Isto ocorre primariamente pela localização do tumor, levando a maiores efeitos sobre o sistema digestório, e também pelos efeitos tóxicos do tratamento quimioterápico ${ }^{11}$.

Contrário à perda ponderal, pacientes oncológicos, em tratamento com alguns tipos de drogas podem ganhar peso, como por exemplo, os que fazem uso de terapia hormonal, comum no tratamento de câncer de mama, podem levar ao aumento de apetite e retenção hídrica ${ }^{12}$.

Um dos sintomas muito comum causados por medicamentos quimioterápicos é a anorexia. Capaz de causar perda ponderal dificulta o tratamento e diminui a qualidade de vida dos pacientes $^{22}$. A anorexia encontra-se presente em $61,6 \%$ dos pacientes deste estudo, possui relação direta com a perda de peso, levando à desnutrição e caquexia.

Outros sintomas muito comuns encontrados na maioria dos pacientes possuindo a mesma característica desse estudo, é a náusea e a alteração de paladar, as quais foram observadas em $60,6 \%$ e $58,6 \%$ desses sintomas, respectivamente, contribuindo mais uma vez para diminuição na ingestão alimentar.

A disfagia e sua relação com a perda de peso estão relacionadas à dificuldade que o paciente apresenta em deglutir. É muito comum apresentação deste sintoma em pacientes com diagnóstico de câncer de esôfago, comprometendo a ingestão alimentar, conduzindo à desnutrição e à resistência à infecção ${ }^{13}$.

Somando-se os diversos efeitos colaterais dos quimioterápicos com a perda ponderal, encontramos um paciente debilitado, imunossuprimido, apresentando baixa qualidade de vida. Muitas vezes, nessa situação, o tratamento pelo qual está passando o paciente, necessita ser interrompido, causando aumento das células tumorais ${ }^{14}$.

A mucosite, outro sintoma muito comum observado em pacientes em tratamento quimioterápico. Constitui em uma condição resultante da inflamação da mucosa oral ou do trato digestório pela ação das drogas utilizadas ${ }^{11}$. 
Nesse estudo, a mucosite foi observada em $27,3 \%$ dos pacientes, não apresentando relação estatística com a redução do percentual de perda de peso, contrariando a maioria dos trabalhos encontrados na literatura. Esse fato pode estar relacionado ao grau mais brando de mucosite apresentado pelos pacientes o qual não foi capaz de interferir na ingestão alimentar.

Conclui-se que é imprescindível conhecer os efeitos colaterais dos tratamentos antineoplásicos, principalmente os quimioterápicos, para que, com ações preventivas, possam-se minimizar os sintomas, a fim de evitar ou reduzir a depleção do estado nutricional do paciente, responsável por um pior prognóstico.

\section{REFERÊNCIAS}

1. Araujo ES, Duval, PA, Silveira, D.H. Sintomas no câncer do aparelho digestório. Rev. Bras. de Canc. [internet]. 2012 [acesso 2015 abr 04];58(4):639-646. Disponível em: http://www.inca.gov.br/rbc/n_58/v04/pdf/09-artigo-sintomas-relacionados-diminuicao-ingestaoalimentar-pacientes-neoplasia-aparelho-digestorio-atendidos-programa-internacao-domiciliar.pdf

2. Sobreira MJ, Bomeny L, Couto DHN. Complicações gastrintestinais relacionadas ao tratamento quimioterápico antineoplásico. Soc. bras. de nutr. paren e ent. [internet] 2011 [acesso 2017 jun 07];13(37). Disponível em: http://sbnperj.com.br/Arquivos/boletim_37.pdf

3. Ferreira NMLA, Scarpa A, Silva DA. Quimioterapia antineoplásica e nutrição: uma relação complexa. Rev. Eletr. Enf. [internet] 2008 [acesso 2017 jun 07];10(4):1026-34. Disponível em: http://www.fen.ufg.br/revista/v10/n4/v10n4a14.htm

4. Correia M. Inquérito brasileiro de avaliação nutricional hospitalar (IBRANUTRI): metodologia do estudo multicêntrico. Rev. Bras. Nutr. Clin. [internet] 1998 [acesso 2016 jun 14];13(1):30-40. Disponível em: http://bases.bireme.br/cgibin/wxislind.exe/iah/online/?IsisScript=iah/ iah.xis\&nextAction=Ink\&base=LILACS\&exprSerch=314600\&indexSearh=ID\&lang=p

5. Tartari RF, Busnello FM, Nunes CHA. Perfil nutricional de pacientes em tratamento quimioterápico em um ambulatório especializado em quimioterapia. Rev bras de canc. [inernet] 2010 [acesso 2015 maio 11]; 56(1):43-50. Disponível em: http://www.inca.gov.br/rbc/n_56/v01/pdf/07_artigo_perfil_nutricional_paciente_oncologico.pdf

6. Poltronieri TS, Tussat C. Impacto do tratamento do câncer sobre o estado nutricional de pacientes oncológicos: atualização da literatura. Revi Bras de Ciên da Saú. 2014;20(4):327-332. DOI: 10.4034/RBCS.2016.20.04.10, https://doi.org/10.4034/RBCS.2016.20.04.10.

7. Nascimento FSM, Gois DNS, Almeida DS, Nascimento AL, Almeida TC, Guedes VR. A importância do acompanhamento nutricional no tratamento e na prevenção do câncer. Ciências Biológicas e de Saúde Unit. [internet] 2015 [acesso 2017 jun 07];2(3):11-24. Disponível em: https://periodicos.set.edu.br/index.php/cadernobiologicas/article/view/1787/1183

8. WHO. World Health Organization. Obesity Preventing and managing the global epidemic: report of a WHO Consultation on Obesity. Geneva: WHO; 1998.

9. LIPSCHITZ, D. A. Screening for nutritional status in the elderly. Prim Care. 1994;22:55-67. 
10. Blackburn, G. L., Bistrian, B.R. Nutritional and metabolic assessment of the hospitalized patient. JPEN, 1: 11-22, 1977, https://doi.org/10.1177/014860717700100101

11. Miranda MP, Souza DS. Glutamina na prevenção e tratamento da mucosite em pacientes adultos oncológicos. Revista Brasileira de Cancerologia. [internet] 2015 [acesso 2017 jun 15];1(3):277-285. Disponível em: http://www.inca.gov.br/rbc/n_61/v03/pdf/11-revisaoglutamina-na-prevencao-e-tratamento-da-mucosite-em-pacientes-adultos-oncologicos-umarevisao-sistematica-da-literatura.pdf.

12. Araújo SNM, Luz MHBA, Silva GRFS, Andrade EMLR, Nunes ICC, Moura RO. O paciente oncológico com mucosite oral: desafios para o cuidado de enfermagem. Rev Lat-Am de Enf. 2015;23(2):267-74. DOI: http://dx.doi.org/10.1590/0104-1169.0090.2551.

13. Barreiro TD. Capacidade alimentar com parâmetro auxiliar do estado nutricional em pacientes com câncer no trato gastrointestinal [dissertação de mestrado]. Porto Alegre: Universidade Federal do Rio Grande do Sul, 2017. Disponível em: https://www.lume.ufrgs.br/bitstream/handle/10183/158272/001020422.pdf?sequence=1

14. Rasia MA, Rosanelli CLSP, Loro M, Gomes JS. Oliveira KR, Kolankiewicz ACB. Efeitos Colaterais de Terapia imunossupressora na percepção de pacientes oncologicos. J Nurs UFPE on line [internet] 2014 [acesso 2016 maio 30];8(4):850-6. Disponível em: http://www.revista.ufpe.br/revistaenfermagem/index.php/revista/article/view/4046/pdf_4849 